\title{
Über Glykosaminoglykane (Mukopolysaccharide) in einem Fall von Lungenfibrose
}

\author{
Atsunobu Yokosawa, Hideo Arai, Hirosi Sato, Masahiko \\ Endo,* Masakichi Motomiya und Kiyoshi Konno \\ Forschungsinstitut für Tuberkulose, Lepra und Krebs, und \\ Medizinisch-Chemisches Institut, Medizinische Fakultät, \\ Tohoku Universität, Sendai
}

Yokosawa, A., Arai, H., Sato, H., Endo, M., Motomiya, M. and Konno, K. On Glycosaminoglycans (Mucopolysaccharide) in a Case of Pulmonary Fibrosis. Tohoku J. exp. Med., 1975, 115 (2), 129-135—Glycosaminoglycans from a case of pulmonary fibrosis (desquamative interstitial pneumonia of usual type) was analyzed by means of proteolytic digestion, solvent fractionation, column chromatography on anion exchanger, electrophoresis and enzymatic digestion. The result suggested an increase in dermatan sulfate and possibly of heparan sulfate in the fibrotic lung as compared with the normal. It is preferable to take the samples for analysis from a freshly excised specimen of the lung, because there was a sign of degradation of glycosaminoglycan due to storage of the specimen in a solution of formalin. - glycosaminoglycan, pulmonary fibrosis

Wie aus den bisherigen Untersuchungen bekannt ist (Koizumi u.a. 1967), nimmt die Menge der Mukopolysacchariden bei der fibrotischen Veränderung des Leberparenchyms zu. Der Zusammenhang zwischen Lungenfibrose und rezidivierenden Entzündungen (Pokorny und Hellwig 1955), Bestrahlung (Bässler und Buchwald 1966), Arzneimittelgabe (Heard 1962) oder Kollagenose (Patterson u.a. 1965) ist oft diskutiert worden. Solche Arbeiten wurden hauptsächlich aus klinischer Sicht veröffentlicht. Im Gegensatz dazu sind die Ergebnisse der systematischen Analyse der Mukopolysaccharide bei der Fibrose der Menschenlunge bis auf die Arbeit von Wusteman u.a. (1972) wenig bekannt.

In der vorliegenden Arbeit werden die Ergebnisse der chemischen Analyse der Mukopolysaccharide (Glykosaminoglykane) bei einer 54-jährigen Patientin mit Lungenfibrose (Pneumonitis interstitialis desquamativa vom gewöhnlichen Typus) beschrieben.

\section{Methodik}

Proteolytischer Abbau und Extraktion des Rohpolysaccharids. Eine in der Formalinlösung aufbewahrte fibrotische Lunge wurde von den großen Bronchien befreit, homogenisiert und in 5 Teilen Äthanol 10 Minuten lang unter Rückfluß auf einem Wasserbad extrahiert. Dieses Verfahren der Äthanolextraktion wurde noch zweimal wiederholt. Anschließend wurde das so entfettete Lungengewebe in eine $0,1 \mathrm{M}$ Calciumacetatlösung $(\mathrm{pH} 8,0)$

Received for publication, October 14, 1974. 
aufgenommen und mit Pronase unter Zugabe von Kanamycin, Toluen und Benzoesäure für 72 Stunden bei $37^{\circ} \mathrm{C}$ inkubiert. Der Reaktionsansatz bekam dann 12-stündlich eine Nachgabe von $2-3 \mathrm{mg}$ pro g Gewebe Pronase. Gleichzeitig wurde das $\mathrm{pH}$ auf 8,0 mit $\mathrm{Ca}(\mathrm{OH})_{2}$-Lösung eingestellt (Arai 1972). Der Reaktionsansatz wurde dann 2 Tage gegen Wasser dialysiert. Der Dialysenrückstand wurde einigermaßen eingeengt und wie oben beschrieben nochmals mit Pronase inkubiert.

Nach dieser Inkubation wurde Trichloressigsaüre in einer Konzentration von 7\% hinzugefügt. Der Ủberstand wurde 2 Tage gegen Wasser dialysiert, eingeengt und bekam 4 Teile mit $\mathrm{NaCl}$ gesättigten $\ddot{A}$ thanol. Der so gewonnene Niederschlag wurde in einer Calciumacetat-Pufferlösung bei pH 8,0 aufgenommen. Die Inkubation mit Pronase, EnteiweiBung mit Trichloressigsäure, und Dialyse gegen Wasser wurden wiederholt. Dann erfolgte die Fällung der Rohpolysaccharid enthaltenden Fraktion durch Zugabe von 4 Teilen Äthanol. Der endgültige Niederschlag wurde mit A thanol und $\ddot{A}$ ther gewaschen und in einem Exsiccator getrocknet (Rohpolysaccharid). Als Kontrolle wurde das Rohpolysaccharid aus den Lungen von drei Patientinnen des gleichen Alters gewonnen, in denen man keine pathologischen Befunde nachweisen konnte, durch ganz gleiche Fraktionierung wie mit der fibrotischen Lunge.

Quantitative Analyse. Uronsäure wurde nach der Vorschrift von Bitter und Muir (1962) bestimmt. Die Bestimmung des Hexosamins erfolgte nach einer modifizierten Vorschrift von Elson-Morgan (Gardell 1953). Die Bestimmung des Neutralzuckers erfolgte nach der Vorschrift von Duboi u.a. (1956) und die des Proteins nach der Vorschrift von Lowry u.a. (1951).

Säulenchromatographische Auftrennung des Rohpolysaccharids. Das Rohpolysaccharid (50 mg) wurde auf eine $2 \times 30 \mathrm{~cm}$ lange Säule von Dowex-1 (Cl-Form) aufgetragen. Elution erfolgte durch um $0,25 \mathrm{M}$ stufenweise gesteigerten Kochsalzgradient ab $0,25 \mathrm{M}$ bis $2,0 \mathrm{M} \mathrm{NaCl}$ und dann mit 5,0 M NaCl (Schiller u.a. 1961). Der Nachweis der Fraktionen erfolgte durch die Bestimmung der Uronsäure. Das Eluat bei jeder verschiedenen Kochsalzkonzentration wurde getrennt gegen Wasser dialysiert, eingeengt und gefriergetrocknet. Anschließend wurden diese Fraktionen durch Elektrophorese auf Celluloseacetatmembran und durch Inkubation mit Mukopolysaccharide-Lyasen qualitativ untersucht.

Elektrophorese. Elektrophorese erfolgte 40 Minuten lang auf einem Cellulosea cetatmembran (Separax, Joko-Sangyo) in einem Gemisch von Ameisensäure und Pyridin bei pH 3,0 (Mathews 1961). Die Stromstärke war $1 \mathrm{~mA} / \mathrm{cm}$. Nach der elektrophoretischen Auftrennung wurde das Elektrophoretogramm in einer $0,05 \%$ Toluidinblaulösung angefärbt.

Inkubation mit Mukopolysaccharide-Lyasen. Die auf die oben geschilderte Weise säulenchromatographisch aufgetrennten Mukopolysaccharide wurden mit Hyaluronidase aus Streptomyces hyalurolyticus bei pH 5,0 in einer Acetatpufferlösung für 1 Stunde bei $60^{\circ} \mathrm{C}$ inkubiert (Ohya und Kaneko 1970). Der Reaktionsansatz mit Chondroitinase AC und der mit Chondroitinase ABC wurden bei pH 8,0 in einer Tris-HCl-Pufferlösung über Nacht bei $37^{\circ} \mathrm{C}$ inkubiert (Yamagata u.a. 1968).

\section{Ergebnisse und Diskussion}

\section{Quantitative Analyse des Rohpolysaccharids (Tabelle 1)}

Der Gehalt an Neutralzucker war höher in der normalen als in der fibrotischen Lunge. Diese Ergebnisse sind verständlich, wenn man das Vorhandensein größerer Mengen von Glykogen in der normalen als in der fibrotischen Lunge annimmt. Der Proteinanteil war höher $(32 \%)$ in der fibrotischen als in der normalen Lunge, was auf die Beteiligung des pronase-resistenten Proteins zurückzuführen ist. Der Gehalt der Uronsäure und Hexosamin war etwas höher in der fibrotischen als in der 
TABELLE 1. Ergebnis der Bestimmung von chemischen Bestandteilen in der Rohpolysaccharidfraktion

\begin{tabular}{|c|c|c|c|c|c|c|c|}
\hline & $\begin{array}{l}\text { Eingesetzte } \\
\text { Menge } \\
\text { (Formalin mit } \\
\text { Filtrierpapier } \\
\text { abgesaugt) } \\
\text { (g) }\end{array}$ & $\begin{array}{c}\text { Entfet- } \\
\text { tetes } \\
\text { Gewebe } \\
\text { (g) }\end{array}$ & $\begin{array}{c}\text { Rohpolysac- } \\
\text { charid } \\
\text { (mg) }\end{array}$ & $\begin{array}{c}\text { Uronsäure } \\
\qquad(\%)\end{array}$ & $\begin{array}{c}\text { Neutral- } \\
\text { zucker }^{b} \text { - } \\
(\%)\end{array}$ & $\begin{array}{c}\text { Hexos- } \\
\operatorname{amin}^{c 1} \\
(\%)\end{array}$ & $\begin{array}{c}\text { Protein }^{\mathrm{d}} \text { ) } \\
(\%)\end{array}$ \\
\hline $\begin{array}{l}\text { Fibrotische } \\
\text { Lunge }\end{array}$ & 56,5 & 12,1 & 151 & 12,0 & 4,6 & 14,0 & 34,8 \\
\hline $\begin{array}{c}\text { Normale } \\
\text { Lunge }\end{array}$ & 56,5 & 6,7 & 116 & 20,0 & 10,7 & 14,9 & 14,9 \\
\hline
\end{tabular}

Bestimmung: a) Bitter und Muir; b) Phenol- $\mathrm{H}_{2} \mathrm{SO}_{4}$; c) Elson-Morgan; d) Lowry.

normalen Lunge. Es ist auch möglich, daß eine größere Menge von Polysaccharid sich in die Formalinlösung während der Zeit des Aufbewahrens aufgelöst hat.

Identifizierung der Mukopolysaccharide aus den säulenchromatographisch aufgetrennten Fraktionen

1. Normale Lunge (Abbildung 1-a)

Die 0,5 M-NaCl Fraktion, die nach der Inkubation mit Hyaluronidase aus Streptomyces hyalurolyticus verschwand, wurde als Hyaluronsäure identifiziert. Zwei Banden in der 0,75 M-NaCl Fraktion wanderten etwas langsamer als Chondroitinsulfat A. Die langsamer wandernde, die nach der Inkubation mit Hyaluronidase aus Streptomyces hyalurolyticus verschwand, wurde als Hyaluronsäure identifiziert. Die schneller wandernde verschwand nach der Inkubation mit Chondroitinase $\mathrm{AC}$ und wurde daher als untersulfatiertes Chondroitinsulfat $\mathrm{A}(\mathrm{C})$ identifiziert. Die 1,0 M-NaCl Fraktion, die auf dem Elektrophoretogramm hinter dem Chondroitinsulfat A wanderte, blieb unverändert nach der Inkubation mit Hyaluronidase aus Streptomyces hyalurolyticus, Chondroitinase AC oder Chondroitinase ABC.

Es wurde also gezeigt, daß diese Fraktion wahrscheinlich Heparansulfat enthielt. Zwei Banden in der 1,25 M-NaCl Fraktion wanderten langsamer als Chondroitinsulfat A. Die schneller wandernde blieb unverändert nach der Inkubation mit Hyaluronidase aus Streptomyces hyalurolyticus oder Chondroitinase AC. Aber sie verschwand nach der Inkubation mit Chondroitinase $A B C$ und wurde daher als Dermatansulfat identifiziert. Die langsamer wandernde, die nach der Inkubation mit Hyaluronidase aus Streptomyces hyalurolyticus, Chondroitinase AC oder Chondroitinase $\mathrm{ABC}$ unverändert blieb, wurde als Heparansulfat identifiziert. Die 1,5 M-NaCl Fraktion zeigte zwei Banden, die auf dem Elektrophoretogramm etwas langsamer als Chondroitinsulfat A wanderten.

Die schneller wandernde blieb unverändert nach der Inkubation mit Hyaluronidase aus Streptomyces hyalurolyticus und verschwand nach der Inkubation mit Chondroitinase AC. Sie wurde daher als untersulfatiertes Chondroitin- 


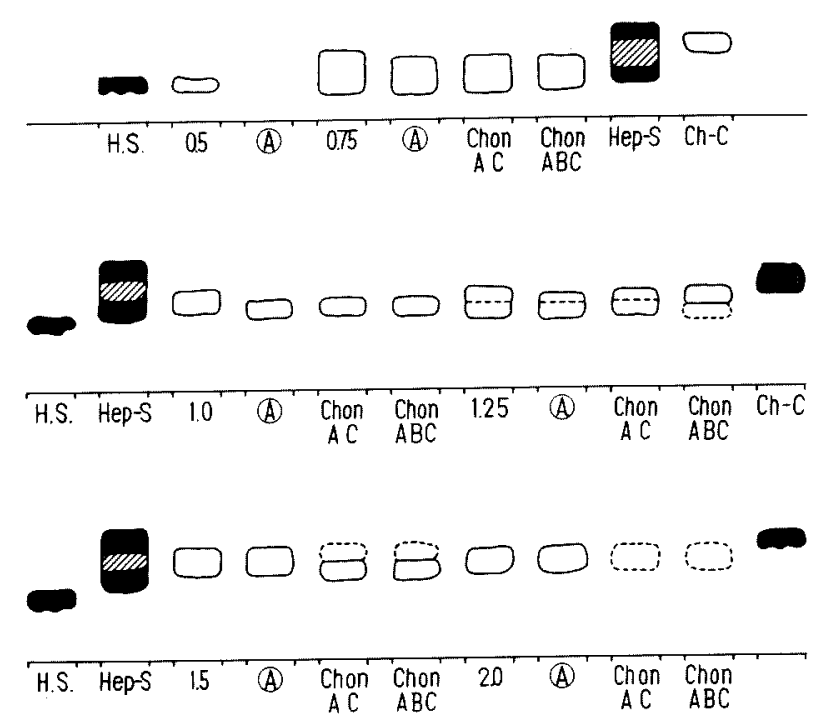

Abb. $1-\mathrm{a}$
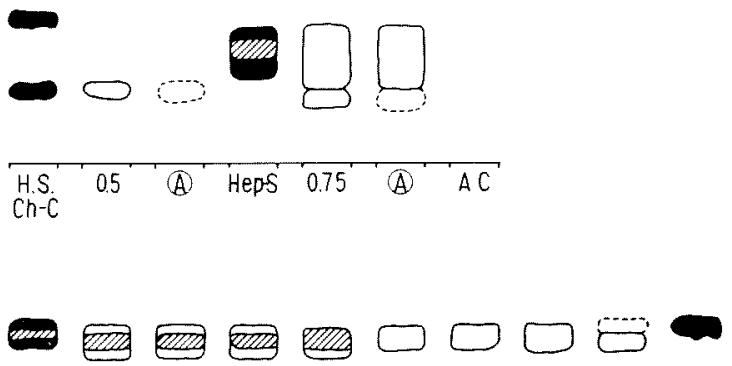

HepS 1.0 (A) $A C \quad A B C \quad 1.25$ (A) $A C$ ABC Ch-S

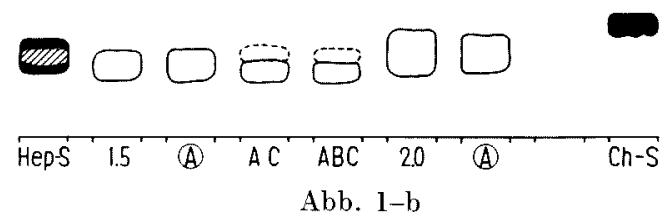

Abb. 1-a Normale Lunge. 1-b Fibrotische Lunge. Elektrophoretogramm auf Celluloseacetatmembran. Puffer, Ameisensäure + Pyridin $(\mathrm{pH}=3,0)$. Stromstärke $1 \mathrm{~mA} / \mathrm{cm}$ 40 min. Anfärbung mit Alcianblau. A, Nach der Inkubation mit Hyaluronidase aus Streptomyces hyalurolyticus; Chon AC, Nach der Inkubation mit Chondroitinase AC; Chon ABC, Nach der Inkubation mit Chondroitinase ABC; H.S., Hyaluronsäure aus Nabelstrang des Menschen (Standard); Hep-S, Heparansulfat (Standard, Seikagaku Kogyo); Chs -C, Chondroitin-6-sulfat (Standard, Seikagaku Kogyo); 0,5, 0,5 M-NaCl Fraktion; 0,75, 0,75 M-NaCl Franktion; 1,0, 1,0 M-NaCl Fraktion; 1,25, 1,25 M-NaCl Fraktion; 1,5, 1,5 M-NaCl Fraktion; 2,0, 2,0 M-NaCl Fraktion. 


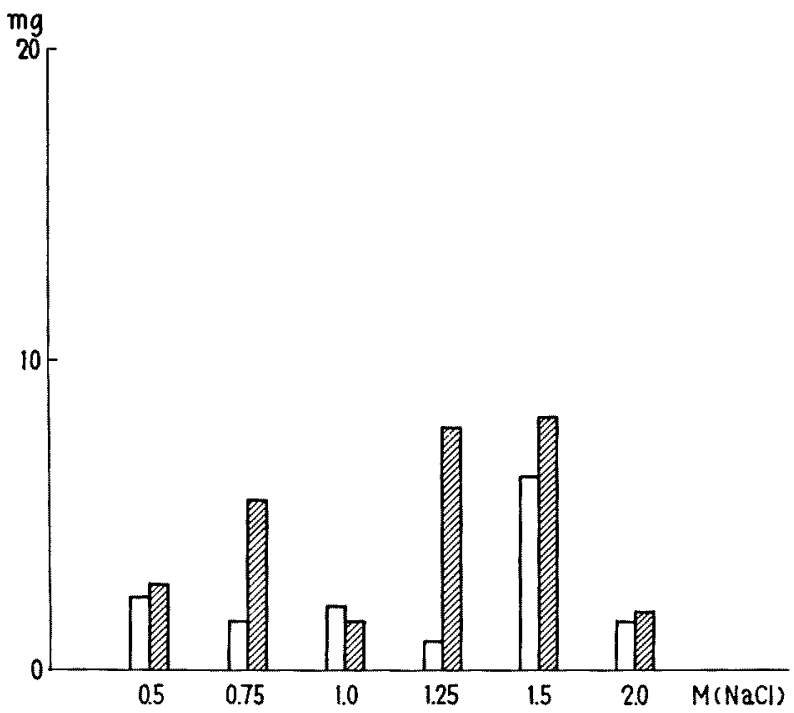

Abb. 2. Menge der Glykosaminoglykane in säulenchromatographisch aufgetrennten Fraktionen. Ergebnis der Gravimetrie. Die weißen Säulen stellen die normale Lunge, die gestrichelten die fibrotische dar.

sulfat $\mathbf{A}(\mathrm{C})$ identifiziert. Die langsamer wandernde, die nach der Inkubation mit Hyaluronidase aus Streptomyces hyalurolyticus, Chondroitinase AC oder Chondroitinase ABC unverändert blieb, wurde als Heparansulfat identifiziert. Die 2,0 M$\mathrm{NaCl}$ Fraktion wanderte etwas langsamer als Chondroitinsulfat $\mathrm{A}(\mathrm{C})$. Sie blieb unverändert nach der Inkubation mit Hyaluronidase aus Streptomyces hyalurolyticus und verschwand nach der Inkubation mit Chondroitinase AC. Daher wurde die 2,0 M-NaCl Fraktion als untersulfatiertes Chondroitinsulfat $\mathrm{A}(\mathrm{C})$ identifiziert.

2. Fibrotische Lunge (Abbildung 1-b)

Die 0,5 M-NaCl Fraktion aus der fibrotischen Lunge verschwand auf dem Elektrophoretogramm nach der Inkubation mit Hyaluronidase aus Streptomyces hyalurolyticus. Diese Bande wurde daher als Hyaluronsäure identifiziert. Die 0,75 M-NaCl Fraktion und die 1,0 M-NaCl Fraktion wanderten etwas langsamer als Chondroitinsulfat A, das als Standard diente. Diese beiden Fraktionen blieben unverändert nach der Inkubation mit Hyaluronidase aus Streptomyces hyalurolyticus, Chondroitinase AC oder Chondroitinase ABC. Diese Untersuchungsergebnisse könnten darauf hinweisen, daß es sich um Heparansulfat handelte. Die 1,25 M-NaCl Fraktion hatte zwei aufeinanderliegende Banden. Die langsamer wandernde verschwand erst nach der Inkubation mit Chondroitinase ABC. Die schneller wandernde blieb unverändert nach der Inkubation mit Hyaluronidase aus Streptomyces hyalurolyticus, Chondroitinase AC oder Chondroitinase ABC.

So wurde festgestellt, daß die 1,25 M-NaCl Fraktion Heparansulfat und Dermatansulfat enthielt. 
Die 1,5 M-NaCl Fraktion hatte auch zwei Banden, die auf dem Elektrophoretogramm hinter Chondroitinsulfat A wanderten. Die schneller wandernde blieb unverändert nach der Inkubation mit Hyaluronidase aus Streptomyces hyalurolyticus. Sie verschwand nach der Inkubation mit Chondroitinase AC. Deshalb wurde sie als untersulfatiertes Chondroitinsulfat $A(C)$ identifiziert. Die langsamer wandernde, die nach der Inkubation mit Hyaluronidase aus Streptomyces hyalurolyticus, Chondroitinase $\mathrm{AC}$ oder Chondroitinase $\mathrm{ABC}$ unverändert blieb, wurde als Heparansulfat identifiziert. Die 2,0 M-NaCl Fraktion wanderte hinter Chondroitinsulfat A. Sie blieb unverändert nach der Inkubation mit Hyaluronidase aus Streptomyces hyalurolyticus und verschwand nach der Inkubation mit Chondroitinase AC. Also wurde die 2,0 M-NaCl Fraktion als untersulfatiertes Chondroitinsulfat $\mathrm{A}(\mathrm{C})$ identifiziert.

Wie aus der Abbildung 2 ersichtlich ist, betrug die 0,5 M-NaCl Fraktion 2,8 $\mathrm{mg}$ in der fibrotischen Lunge und $2,4 \mathrm{mg}$ in der normalen Lunge. Der Unterschied in der Menge bei der 1,0 M-NaCl Fraktion war nur 0,5 mg (fibrotische Lunge - 1,6 mg, normale Lunge - 2,1 mg). Dagegen waren die Mengen der $0,75 \mathrm{M}-\mathrm{NaCl}$ und 1,25 $\mathrm{M}-\mathrm{NaCl}$ Fraktionen weit größer in der fibrotischen Lunge als die der entsprechenden Fraktionen in der normalen Lunge.* So dürfte man annehmen, daß die Menge des Heparansulfats und die des Dermatansulfats in der fibrotischen Lunge im Vergleich mit der normalen zunehmen. Die Anwesenheit größerer Mengen untersulfatierten Chondroitinsulfats und der sehr niedrige Gehalt an Neutralzucker in der Lunge, die in der Formalinlösung aufbewahrt wurde, könnte darauf hinweisen, daß die Mukopolysaccharide sich mit der Zeit des Aufbewahrens zersetzen oder daß die Polysaccharide sich in die Formalinlösung auflösen. Ein weiterer Versuch mit frisch gewonnenen Lungen ist jetzt im Gang. ichtet.

Wir sind Herrn Professor Z. Yosizawa für seine Leitung zum herzlichsten Dank verpfl-

\section{Literatur}

1) Arai, H. (1972) A study on acidic mucopolysaccharides in pleural and peritoneal effusions, and in ascites. Sci. Rep. Res. Inst. Tohoku Univ., Ser. C, 19, 26-37.

2) Bässler, R. \& Buchwald, W. (1966) Experimentelle Entzündung und Fibrose des Lungengerüstes durch ionisierende Strahlen. Licht- und elektronenmikroskopische Untersuchungen. Fortschr. Röntgenstr., 104, 192-206.

3) Bitter, T. \& Muir, H.M. (1962) A modified uronic acid carbazole reaction. Analyt. Chem., 4, 330-334.

4) Duboi, M., Gilles, K.A., Hamilton, J.K., Rebers, P.A. \& Smith, F. (1956) Colorimetric method for determination of sugars and related substances. Analyt. Chem., 28, 350-356.

5) Gardell, S. (1953) Separation on Dowex-50 ion exchange resin of glucosamine and galactosamine and their quantitative determination. Acta chem. scand., 7, 207-215.

* Die 0,75 M-NaCl Fraktion aus der fibrotischen Lunge enthielt Heparansulfat und die der normalen Lungen Hyaluronsäure sowie untersulfatiertes Chondroitinsulfat $A(C)$. Die 1,25 M-NaCl Fraktionen aus den fibrotischen und normalen Lungen enthielten Heparansulfat sowie Dermatansulfat. Die 1,5 M-NaCl Fraktionen aus den fibrotischen und normalen Lungen enthielten Heparansulfat sowie untersulfatiertes Chondroitinsulfat $A(C)$. 
6) Heard, B.E. (1962) Fibrous healing of old iatrogenic pulmonary oedema ("Hexamethonium lung"). J. Path. Bact., 83, 159-164.

7) Koizumi, T., Nakamura, N. \& Abe, H. (1967) Changes in acid mucopolysaccharides in the liver in hepatic fibrosis. Biochim. biophys. Acta (Amst.), 148, 749-756.

8) Lowry, O.H., Rosebrough, N.J., Farr, A.L. \& Randall, R.J. (1951) Protein measurement with the Folin phenol reagent. J. biol. Chem., 193, 265-275.

9) Mathews, M.B. (1961) Acid strength of carboxyl groups in isomeric chondroitin sulfates. Biochim. biophys. Acta (Amst.), 48, $402-403$.

10) Ohya, T. \& Kaneko, Y. (1970) Novel hyaluronidase from streptomyces. Biochim. biophys. Acta (Amst.), 198, 607-609.

11) Patterson, C.D., Harville, W.E. \& Pierce, J.A. (1965) Rheumatoid lung disease. Ann. intern. Med., 62, 685-697.

12) Pokorny, C.H. \& Hellwig, C.A. (1955) Diffuse interstitial fibrosis of the lung. Arch. Path., 59, 382-387.

13) Schiller, S., Slover, G.A. \& Dorfman, A. (1961) A method for the separation of acid mucopolysaccharides: Its application to the isolation of heparin from the skin of rats. J. biol. Chem., 236, 983-987.

14) Wusteman, F.S., Gold, C. \& Wagner, J.C. (1972) Glycosaminoglycans and calcification in the lesion of progressive massive fibrosis and in pleural plaque. Amer. Rev. resp. Dis., 106, 116-118.

15) Yamagata, T., Saito, H., Habuchi, H. \& Suzuki, S. (1968) Purification and properties of bacterial chondroitinases and chondrosulfatases. J. biol. Chem., 243, 1523-1535. 